\title{
Usefulness of serum D-dimer for preoperative diagnosis of infected nonunion after open reduction and internal fixation
}

This article was published in the following Dove Press journal:

Infection and Drug Resistance

\author{
Zhen Wang ${ }^{1, *}$ \\ Chong Zheng ${ }^{2, *}$ \\ Siyuan Wen' \\ Junfei Wang' \\ Zitao Zhang' \\ Xusheng Qiu' \\ Yixin Chen'
}

'Department of Orthopedics, Drum Tower Hospital Clinical College of Nanjing University, Nanjing, People's Republic of China; ${ }^{2}$ Department of Orthopedics, Changzhou Traditional Chinese Medicine Hospital Affiliated to Nanjing University of Chinese Medicine, Changzhou, People's Republic of China

*These authors contributed equally to this work
Correspondence: Yixin Chen Department of Orthopedics, Drum Tower Hospital Clinical College of Nanjing University, 32I Zhongshan Road, Nanjing, Jiangsu 210008, People's Republic of China

Email chenyixin93@126.com
Purpose: Infected nonunion after open reduction internal fixation (ORIF) is a serious complication. The aim of this study was to evaluate the usefulness of serum D-dimer for preoperative diagnosis of infected nonunion.

Patients and methods: Patients undergoing debridement and external fixation for infected nonunion $(n=32)$ and replacement of internal fixation due to aseptic failure $(n=34)$ were enrolled and compared in this retrospective study. The optimum cutoff value of D-dimer for identification of infected nonunion was determined by calculating the Youden $\mathrm{J}$ statistic. The sensitivity, specificity, positive predictive value (PPV), and negative predictive value (NPV) of four preoperative laboratory parameters - serum D-dimer level, white blood cell (WBC) count, erythrocyte sedimentation rate (ESR), and C-reactive protein (CRP) - for diagnosis of infected nonunion were compared.

Results: Serum D-dimer level was significantly higher in patients with infected nonunion than in patients with aseptic nonunion: $2.62 \mathrm{mg} / \mathrm{mL}$ (range, $0.13-11.90 \mathrm{mg} / \mathrm{mL}$ ) vs $0.35 \mathrm{mg}$ / $\mathrm{mL}$ (range, $0.07-6.46 \mathrm{mg} / \mathrm{mL} ; p<0.001$ ). WBC count, CRP, and ESR demonstrated sensitivity of $12.5 \%$ (95\% CI: 4.08-29.93), 40.6\% (95\% CI: 24.22-59.21), and 56.3\% (95\% CI: $37.88-73.16$ ), respectively, and specificity of $94.1 \%$ (95\% CI: 78.94-98.97), 88.2\% (95\% CI: 71.61-96.16), and 85.3\% (95\% CI: 68.17-94.46), respectively. Using the Youden index, $1.70 \mathrm{mg} / \mathrm{mL}$ was determined as the optimal threshold value for serum D-dimer for the diagnosis of infected nonunion. The sensitivity and specificity of serum D-dimer (>1.70 mg/mL) were 75.0\% (95\% CI: 56.25-87.87) and 91.2\% (95\% CI: 75.19-97.69).

Conclusions: Serum D-dimer level may be useful for preoperative prediction of infected nonunion in patients after ORIF.

Keywords: fracture-related infection, laboratory test, preoperative prediction, nonunion

\section{Introduction}

Infected nonunion after open reduction and internal fixation (ORIF) is a major challenge for orthopedic surgeons. If not promptly diagnosed and managed, permanent loss of function, amputation, and even death may result. ${ }^{1}$ However, in the absence of any reliable method for diagnosing infection prior to nonunion surgery, surgeons cannot accurately discriminate between infected nonunion and aseptic nonunion. ${ }^{8,9}$ The Association for Osteosynthesis/Association for the Study of Internal Fixation (AO/ASIF) consensus definition of fracture-related infection includes four confirmatory criteria and six suggestive criteria. ${ }^{2}$ While positive culture of intraoperative specimen remains the gold standard for diagnosis, it is 
time-consuming and has relatively poor sensitivity. ${ }^{3}$ Currently, evaluation of inflammatory markers is the first step in a fracture nonunion patient with clinically suspected infection. ${ }^{4}$ White blood cell (WBC) count, erythrocyte sedimentation rate (ESR), and $\mathrm{C}$-reactive protein (CRP) have traditionally been used as screening tests for infection because of their simplicity and costeffectiveness. ${ }^{5,6}$ However, the sensitivity and specificity of these tests have declined in recent years because of the decrease in the number of patients with typical clinical manifestations of infection. ${ }^{4,7}$ It has therefore become essential to identify additional laboratory tests that can help in preoperative diagnosis.

D-dimer - a fibrin degradation product in plasma - was widely used earlier as a diagnostic aid in suspected venous thromboembolism (VTE) and pulmonary embolism (PE), but has now largely been abandoned because of its poor specificity. ${ }^{10-12}$ Recently, some studies have shown that systemic and local infections result in increased fibrinolytic activity and raise serum D-dimer levels. ${ }^{13-16}$ Different groups have also demonstrated that the D-dimer level is a predictor of poor outcome in sepsis, bacteremia, and periprosthetic joint infection (PJI). ${ }^{17-19}$

The aim of this study was to investigate whether the D-dimer level could be used for preoperative diagnosis of infected nonunion after ORIF.

\section{Materials and methods}

\section{Patients}

A total of 108 consecutive patients treated operatively for primary nonunion between March 2016 and December 2018 were screened for eligibility for inclusion in this retrospective study. The inclusion criteria were as follows: 1) patients aged $\geq 18$ years; 2) those with nonunion that required primary operation. Patients were excluded if they 1) had received antibiotics before surgery; 2) had prosthetic heart valve implant or any type of skin ulcer, hematoma, or visible ecchymosis; 3) had history of any hypercoagulation disorder (eg, VTE, PE, or disseminated intravascular coagulation); or 4) had sepsis or infections not involving the fracture site. A total of 66 patients met these criteria and were included for analysis. The patients were separated into two groups as follows: 32 in Group A (revision for infected nonunion) and 34 in Group B (revision for aseptic nonunion).

Ethical approval was obtained from the Clinical Research Ethics Committee of The Affiliated Drum Tower
Hospital of Nanjing University Medical School. All participants consented to their data being used for research.

Data were collected on baseline demographics (ie, sex and age); body mass index, smoking history, the involved location; results of blood tests on admission to hospital (ie, serum D-dimer, WBC count, CRP, and ESR); and blood culture results. To prevent the occurrence of PE, D-dimer is routinely evaluated to diagnose DVT in all patients at admission in our department. The definition of a long-bone nonunion was "radiographic evidence of nonprogression of healing for at least 3 months, or lack of healing by 9 months since the initial injury. Infected nonunion was defined using the AO/ASIF criteria. ${ }^{2}$ A positive diagnosis of infection was made if the same organism was grown in at least two cultures of the intraoperative sample.

\section{Statistical analysis}

For demographic characteristics, continuous variables are presented as mean $\pm \mathrm{SD}$ and categorical variables as absolute numbers and proportions. The chi-squared test was used to analyze categorical data, and Student's $t$-test was used to analyze continuous variables. All laboratory values were summarized as median. The Mann-Whitney $U$ test was used to compare the results between the groups. The optimal threshold value of D-dimer for diagnosis of infected nonunion was determined by calculating the Youden $\mathrm{J}$ statistic $(\mathrm{J}=[$ sensitivity + specificity] -1$)$. The sensitivity, specificity, positive predictive value (PPV), and negative predictive value (NPV) of WBC count, CRP, ESR, and D-dimer were calculated. The $95 \%$ CIs were calculated according to the efficient-score method. ${ }^{20}$ All statistical analyses were performed using STATA version 18.0 (SPSS Inc., Chicago, IL, USA). Statistical significance was at $p<0.05$.

\section{Results}

A total of 66 patients ( 9 women and 57 men; mean age, $57.0 \pm 11.8$ years; age range, $18-71$ years) were enrolled in this study. Table 1 summarizes the demographic characteristics of patients in the two groups.

Among those with infected nonunion, 13/32 had methicillin-sensitive Staphylococcus aureus infection, 4/32 had methicillin-resistant $S$ aureus infection, 5/32 had Staphylococcus epidermidis infection, 2/32 had Escherichia coli infection, 1/32 had Enterobacter cloacae infection, 1/32 had Enterococcus faecalis infection, 1/32 
Table I Demographics of the two groups

\begin{tabular}{|l|l|l|l|}
\hline & $\begin{array}{l}\text { Group A } \\
(\mathbf{n = 3 2 )}\end{array}$ & $\begin{array}{l}\text { Group B } \\
(\mathbf{n = 3 4 )}\end{array}$ & $\boldsymbol{P}$-value \\
\hline No. of women & $2 / 30$ & $7 / 27$ & 0.090 \\
Age (year, mean \pm SD) & $45.5 \pm 14.7$ & $43.9 \pm 12.8$ & 0.654 \\
BMI (kg/m², mean \pm SD) & $23.5 \pm 3.8$ & $23.3 \pm 4.1$ & 0.832 \\
Current smoker (yes/no) & $14 / 18$ & $13 / 21$ & 0.649 \\
Cancer (yes/no) & $2 / 30$ & $1 / 33$ & 0.519 \\
Vascular disease (yes/no) & $3 / 29$ & $4 / 30$ & 0.753 \\
Nonunion site (lower extremity, yes/no) & $26 / 6$ & $27 / 7$ & 0.851 \\
\hline
\end{tabular}

Notes: Group A = infected nonunion; Group $B=$ aseptic nonunion. $P<0.05$ indicate significance.

Abbreviation: $\mathrm{BMI}$, body mass index.

Table 2 Comparison of blood parameters between two groups

\begin{tabular}{|l|l|l|l|}
\hline & $\begin{array}{l}\text { Group A } \\
(\mathbf{n = 3 2})\end{array}$ & $\begin{array}{l}\text { Group B } \\
(\mathbf{n = 3 4 )}\end{array}$ & P-value \\
\hline WBC $\left(10^{9} / \mu \mathrm{L}\right.$, median) & 6.8 & 6.3 & 0.91 \\
CRP $(\mathrm{mg} / \mathrm{L}$, median) & 6.6 & 3.2 & $0.03^{*}$ \\
ESR $(\mathrm{mm} / \mathrm{hr}$, median) & 15.5 & 6.0 & $<0.00 I^{*}$ \\
D-dimer (mg/L, median) & 2.6 & 0.3 & $<0.00 I^{*}$ \\
\hline
\end{tabular}

Notes: Group $A=$ infected nonunion; Group $B=$ aseptic nonunion. $* P<0.05$ indicate significance.

Abbreviations: WBC, white blood cell; CRP, C-reactive protein; ESR, erythrocyte sedimentation rate.

had Streptococcus mutans infection, and 5/32 had polymicrobial infection.

Median serum D-dimer level was significantly higher in the infected nonunion group than in the aseptic nonunion group. Similarly, median ESR and CRP values were also significantly higher in the infected nonunion group than in the aseptic nonunion group. However, the median WBC count was comparable between the two groups. (Table 2)

Table 3 shows the sensitivity and specificity of each of the four tests. WBC count had high specificity $(94.1 \%, 95 \% \mathrm{CI}$ : 78.94-98.97) but the lowest sensitivity $(12.5 \%, 95 \% \mathrm{CI}$ : 4.08-29.93). Similarly, CRP and ESR had low sensitivity of $40.6 \%$ (95\% CI: $24.21-59.21)$ and $56.3 \%$ (95\% CI: $37.88-73.16 \%$ ), respectively, and high specificity of $88.2 \%$ (95\% CI: 71.61-91.16) and 85.3\% (95\% CI: 68.17-94.46), respectively. D-dimer $(>1.70 \mathrm{mg} / \mathrm{mL})$ had the most high specificity (75.0\%, 95\%CI: 56.25-87.87) and better sensitivity (91.2\%, 95\% CI: 75.19-97.69). Both the PPV and NPV of D-dimer were better than the other three tests.

\section{Discussion}

Recently, Gris et al ${ }^{17}$ reported that elevated serum D-dimer predicts poor outcome in septic shock, and Schwameis et al ${ }^{18}$ found that the D-dimer level was a predictor of risk of mortality in the very early stages of bacteremia. In addition, Shahi et $\mathrm{al}^{19}$ showed that serum D-dimer was a useful marker of PJI. However, there have been no studies so far on the utility of D-dimer in the diagnosis of infected nonunion. In this study, we demonstrated that serum D-dimer level $>1.70 \mathrm{mg} / \mathrm{mL}$ has better sensitivity than the other commonly used laboratory tests - WBC, ESR, and CRP - for diagnosis of infected nonunion. Additionally, the specificity of D-dimer was so high among them.

WBC count, CRP, and ESR are the most commonly used markers of inflammation. Changes in these parameters generally indicate the onset of infection. Unfortunately, however, all three tests are affected by factors such as physiological stress, treatment, and trauma. ${ }^{4,21,22}$ In the present study, we found that WBC count had high specificity (94.1\%, 95\% CI: 78.94-98.97), but very low sensitivity (12.5\%, 95\% CI: 4.08-29.93); this finding is consistent with previous studies. ${ }^{7,22}$ CRP and ESR demonstrated only moderate sensitivities and specificities.

D-dimer is a marker of fibrinolysis and was earlier used widely, albeit with disappointing performance, for screening patients for VTE. ${ }^{10-12}$ Recently, a number of studies have proposed that serum D-dimer level is an effective serum inflammatory marker with distinct advantages for the detection of systemic inflammation and infection. ${ }^{14,17,18}$ Kinasewitz et $\mathrm{al}^{23}$ and Deitcher et $\mathrm{al}^{24}$ reported that D-dimer level was a sensitive test for identification of sepsis in intensive care unit patients. Shahi et $\mathrm{al}^{19}$ found that elevated D-dimer level in patients undergoing reimplantation could be an indication of persistent infection. In the present study, patients with infected nonunion had significantly higher serum D-dimer levels than others. D-dimer $(>1.70 \mathrm{mg} / \mathrm{mL})$ had the most high specificity $(75.0 \%, 95 \%$ CI: $56.25-87.87)$ and better sensitivity $(91.2 \%, 95 \%$ CI: 75.19-97.69) than the other tests. 
Table 3 Performance of laboratory tests in the diagnosis of infected nonunion

\begin{tabular}{|l|l|l|l|l|}
\hline & WBC & CRP & ESR & D-dimer \\
\hline False positive, $\mathrm{n}$ & 2 & 4 & 5 & 3 \\
True negative, $\mathrm{n}$ & 32 & 30 & 29 & 31 \\
True positive, $\mathrm{n}$ & 4 & 13 & 18 & 24 \\
False negative, $\mathrm{n}$ & 28 & 19 & 14 & 8 \\
Sensitivity (95\% Cl) & $12.5 \%(4.08-29.93)$ & $40.6 \%(24.21-59.21)$ & $56.3 \%(37.88-73.16)$ & $75.0 \%(56.25-87.87)$ \\
Specificity (95\% Cl) & $94.1 \%(78.94-98.97)$ & $88.2 \%(71.61-91.16)$ & $85.3 \%(68.17-94.46)$ & $91.2 \%(75.19-97.69)$ \\
PPV (95\% Cl) & $66.7 \%(24.11-94.00)$ & $76.5 \%(49.76-92.18)$ & $78.3 \%(55.79-91.71)$ & $88.9 \%(69.70-97.09)$ \\
NPV (95\% Cl) & $53.3 \%(40.10-66.14)$ & $61.2 \%(46.24-74.46)$ & $67.4 \%(51.34-80.46)$ & $79.5 \%(63.06-90.13)$ \\
\hline
\end{tabular}

Abbreviations: WBC, white blood cell; CRP, C-reactive protein; ESR, erythrocyte sedimentation rate; PPV, positive predictive value; NPV, negative predictive value.

Disseminated intravascular coagulation, characterized by circulating fibrinogen degradation products, is associated with inflammatory conditions. Coagulation activation results when endothelial damage leads to exposure of blood to extravascular tissue factors. ${ }^{18}$ Activation of the coagulation cascade is a common and early event in patients with infection, and many of the molecules involved in this process are also important amplifiers of the inflammatory response. ${ }^{25,26}$ Moreover, D-dimer can itself mediate and enhance the inflammatory response. ${ }^{27}$ According to Ribera et al, ${ }^{14}$ D-dimer may help in localizing infecting organisms or inflammatory cells.

This present study has several limitations. First, this was a retrospective observational study and some bias is inevitable. Second, the number of patients in each group was relatively small. Prospective studies with larger samples would give more robust evidence. Finally, in contrast to PJI, these standardized protocols tailored to diagnose infected nonunion in patients after ORIF are scarce. The laboratory serum tests have no confirmatory criteria to diagnose infected nonunion, which may influence our results.

\section{Conclusion}

To our knowledge, this study is the first to indicate that serum D-dimer may have value for diagnosis of infection in bone nonunion patients after ORIF. D-dimer level $>1.70 \mathrm{mg} / \mathrm{mL}$ appears to provide the optimum balance of sensitivity and specificity for the diagnosis of infected nonunion. However, it must be stressed that diagnosis of infected nonunion should always be based on the combined results of clinical, laboratory, and radiologic evaluations.

\section{Abbreviation list}

AO/ASIF, Association for Osteosynthesis/Association for the Study of Internal Fixation; CRP, C-reactive protein;
ESR, erythrocyte sedimentation rate; NPV, negative predictive value; ORIF, open induction internal fixation; PE, pulmonary embolism; PPV, positive predictive value; VTE, venous thromboembolism; WBC, white blood cell.

\section{Acknowledgments}

This work was supported by National Natural Science Foundation of China (Grant no. 81871762).

\section{Disclosure}

The authors report no conflicts of interest in this work.

\section{References}

1. Bonnevialle P. Operative treatment of early infection after internal fixation of limb fractures (exclusive of severe open fractures). Orthop Traumatol Surg Res. 2017;103(1S):S67-s73. doi:10.1016/j.otsr.2016.06.019

2. Metsemakers WJ, Morgenstern M, McNally MA, et al. Fracturerelated infection: a consensus on definition from an international expert group. Injury. 2018;49(3):505-510. doi:10.1016/j.injury.2017. 08.040

3. Barrack RL, Jennings RW, Wolfe MW, Bertot AJ. The coventry award. The value of preoperative aspiration before total knee revision. Clin Orthop Relat Res. 1997;345:8-16.

4. Wang S, Yin P. Evaluating the use of serum inflammatory markers for preoperative diagnosis of infection in patients with nonunions. Biomed Res Int. 2017;2017:9146317.

5. Shin WC, Do MU, Woo SH, Choi SH, Moon NH, Suh KT. C-reactive protein for early detection of postoperative systemic infections in intertrochanteric femoral fractures. Injury. 2018;49(10):1859-1864. doi:10.1016/j.injury.2018.07.029

6. Kim TW, Kim DH, Oh WS, Sim JA, Lee YS, Lee BK. Analysis of the causes of elevated C-reactive protein level in the early postoperative period after primary total knee arthroplasty. $J$ Arthroplasty. 2016;31 (9):1990-1996. doi:10.1016/j.arth.2016.02.037

7. Morgenstern M, Kuhl R, Eckardt H, et al. Diagnostic challenges and future perspectives in fracture-related infection. Injury. 2018;49(Suppl 1):S83-S90. doi:10.1016/S0020-1383(18)30310-3

8. Olszewski D, Streubel PN, Stucken C, et al. Fate of patients with a "Surprise" positive culture after nonunion surgery. J Orthop Trauma. 2016;30(1):e19-e23. doi:10.1097/BOT.0000000000000417

9. Zhang Z, Ji Y, Wang Z, Qiu X, Chen Y. The association between platelet indices and deep surgical site infection after open induction internal fixation for traumatic limb fractures. Infect Drug Resist. 2018;11:2533-2538. doi:10.2147/IDR.S184877 
10. Bounameaux H, de Moerloose P, Perrier A, Reber G. Plasma measurement of D-dimer as diagnostic aid in suspected venous thromboembolism: an overview. Thromb Haemost. 1994;71(1):1-6.

11. Hansrani V, Khanbhai M, McCollum C. The diagnosis and management of early deep vein thrombosis. Adv Exp Med Biol. 2017;906:23-31. doi:10.1007/5584_2016_103

12. Palareti G, Legnani C, Cosmi B, Guazzaloca G, Pancani C, Coccheri S. Risk of venous thromboembolism recurrence: high negative predictive value of D-dimer performed after oral anticoagulation is stopped. Thromb Haemost. 2002;87(1):7-12.

13. Gando S. Role of fibrinolysis in sepsis. Semin Thromb Hemost. 2013;39(4):392-399. doi:10.1055/s-0033-1334140

14. Ribera T, Monreal L, Armengou L, Rios J, Prades M. Synovial fluid D-dimer concentration in foals with septic joint disease. $J$ Vet Intern Med. 2011;25(5):1113-1117. doi:10.1111/j.1939-1676.2011.0758.x

15. Michelin E, Snijders D, Conte S, et al. Procoagulant activity in children with community acquired pneumonia, pleural effusion and empyema. Pediatr Pulmonol. 2008;43(5):472-475. doi:10.1002/ ppul.20795

16. Goebel PJ, Williams JB, Gerhardt RT. A pilot study of the performance characteristics of the D-dimer in presumed sepsis. West J Emerg Med. 2010;11(2):173-179.

17. Gris JC, Bouvier S, Cochery-Nouvellon E, Faillie JL, LissaldeLavigne G, Lefrant JY. Fibrin-related markers in patients with septic shock: individual comparison of D-dimers and fibrin monomers impacts on prognosis. Thromb Haemost. 2011;106(6):1228-1230. doi:10.1160/TH11-07-0489

18. Schwameis M, Steiner MM, Schoergenhofer C, et al. D-dimer and histamine in early stage bacteremia: a prospective controlled cohort study. Eur J Intern Med. 2015;26(10):782-786. doi:10.1016/j. ejim.2015.10.024
19. Shahi A, Kheir MM, Tarabichi M, Hosseinzadeh HRS, Tan TL, Parvizi J. Serum D-Dimer test is promising for the diagnosis of periprosthetic joint infection and timing of reimplantation. $J$ Bone Joint Surg Am. 2017;99(17):1419-1427. doi:10.2106/ JBJS.16.01395

20. Newcombe RG. Two-sided confidence intervals for the single proportion: comparison of seven methods. Stat Med. 1998;17(8):857-872.

21. Ovaska MT, Makinen TJ, Madanat R, Vahlberg T, Hirvensalo E, Lindahl J. Predictors of poor outcomes following deep infection after internal fixation of ankle fractures. Injury. 2013;44 (7):1002-1006. doi:10.1016/j.injury.2013.02.027

22. Metsemakers WJ, Kuehl R, Moriarty TF, et al. Infection after fracture fixation: current surgical and microbiological concepts. Injury. 2018;49(3):511-522. doi:10.1016/j.injury.2016.09.019

23. Kinasewitz GT, Yan SB, Basson B, et al. Universal changes in biomarkers of coagulation and inflammation occur in patients with severe sepsis, regardless of causative micro-organism [ISRCTN74215569]. Crit Care. 2004;8(2):R82-R90. doi:10.1186/cc2459

24. Deitcher SR, Eisenberg PR. Elevated concentrations of cross-linked fibrin degradation products in plasma. An early marker of gram-negative bacteremia. Chest. 1993;103(4):1107-1112. doi:10. 1378/chest.103.4.1107

25. Cicala C, Cirino G. Linkage between inflammation and coagulation: an update on the molecular basis of the crosstalk. Life Sci. 1998;62 (20):1817-1824

26. Cirino G, Cicala C, Bucci MR, Sorrentino L, Maraganore JM, Stone SR. Thrombin functions as an inflammatory mediator through activation of its receptor. $J$ Exp Med. 1996;183(3):821-827. doi:10.1084/jem.183.3.821

27. Tripodi A. D-dimer testing in laboratory practice. Clin Chem. 2011;57(9):1256-1262. doi:10.1373/clinchem.2011.166249
Infection and Drug Resistance

\section{Publish your work in this journal}

Infection and Drug Resistance is an international, peer-reviewed openaccess journal that focuses on the optimal treatment of infection (bacterial, fungal and viral) and the development and institution of preventive strategies to minimize the development and spread of resistance. The journal is specifically concerned with the epidemiology of antibiotic resistance and the mechanisms of resistance development and diffusion in both hospitals and the community. The manuscript management system is completely online and includes a very quick and fair peerreview system, which is all easy to use. Visit http://www.dovepress.com/ testimonials.php to read real quotes from published authors. 